AFCRL-TN-60-687

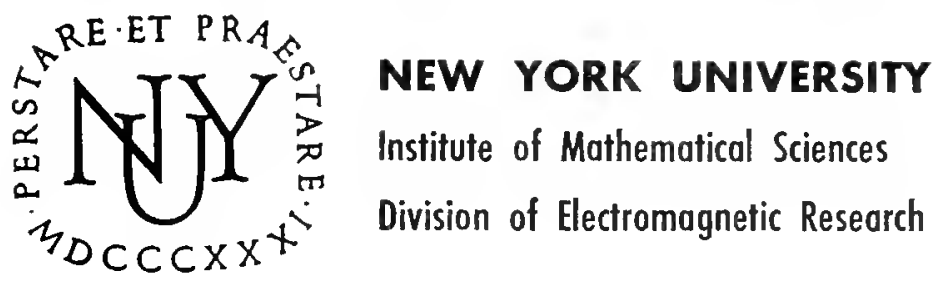

RESEARCH REPORT NO. CX-52

\title{
Bounds on Scattering Phase Shifts For Compound Systems
}

LEONARD ROSENBERG and LARRY SPRUCH

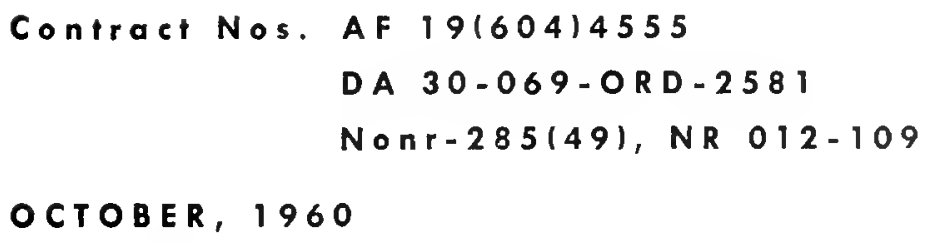


NEW YORK UNIVERSITY

Institute of Mathemat1cal Sciences

Division of Electromagnet1c Research

Research Report No. CX-52

BOUNDS ON SCATTERING PHASE SHIFTS

FOR COMPOUND SYSTEMS

Leonard Rosenberg and Larry Spruch

The research reported in this paper has been jointly sponsored by the Geophysics Research Directorate of the Air Force Cambridge Research Center, Air Research and Development Command, under Contract No. $A F$ 19(604)4555, and the office of Ordnance Research under Contract No. DA30-069-ORD-2581, Project No. 2360, and the Office of Naval Research under Contract Nonr-285(49), NR 012-109.
Leovard Rowenberg

Leonard Rosenberg

harry Spruch

Larry Spruch

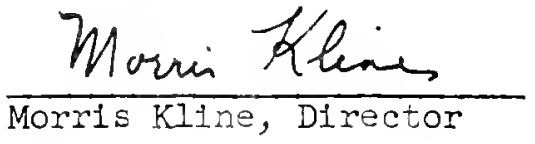


Requests for additional coples by Agencies of the Department of Defense, their contractors, and other Government agencies should be directed to the:

ARMED SERVICES TECHNICAL INFORMATION AGENCY

DOCUMENTS SERVICE CENTER

ARLINGTON HALL STATION

ARLINGTON 12, VIRGINIA

Department of Defense contractors must be established for ASTIA services or have thelr 'need-to-know' certifled by the cognizant military agency of their project or contract. All other persons and organizations apply to the:

U.S. DEPARTMENT OF COMMERCE

OFFICE OF TTECHNICAI SERVICES

WASHINGTON 25, D.C. 
An extension of recently developed methods determines a rigorous upper bound on $(-k \cot \eta)^{-1}$, where $\eta$ is the phase shift, for the general one channel scattering process. The method unfortunately requires truncation of the various potentials, but it should generally be possible, in practice, to so truncate the potentials that the aifference between the phase shifts of the orlginal problem and of the problem for which a bound is obtained is insigniflcant.

In the course of the development it is necessary to introduce, for compound system scattering, an absolute definition of the phase shift, not simply a definition modulo $\pi$. The definition chosen is to take the projection of the full scattering wave function on the ground state wave function of the scattering system, and to treat the resultant one coordinate wave function as if it were the scattering wave function for a particle on a center of force. Though irrelevant with regard to the determination of a bound on cot $\eta$, it is interesting that at least for some simple cases this deflnition automatically increases the phase shift by at least $\pi$ whenever the Pauli prinicple introduces a spatial node into the scattering wave function. The triplet scattering of electrons by $H$ atoms provides an example. 



\section{Table of Contents}

Page

1. Introduction 2

2. Definition of the Phase Shift 5

An Application: Triplet Electron Hydrogen Scattering 6

3. Definition of the Problem for which the Bound is to be Obtained 8

4. Bound on $k \cot (\eta-\theta)$ and on $\eta \quad 11$

A - The Associated Potential Strength Elgenvalue Problem 11

B - The Conditional Inequality $\quad 14$

5. The Use of Energy Eigenfunctions 17

References 22 


\section{1 - INTRODUCTION}

In a series of papers ${ }^{1-4}$, it has been shown that for the one channel problem, to which the present paper is restricted, it is possible to replace certain variational principles for scattering theory by much more powerful minimum principles. More specifically, a rigorous upper bound on the scattering length, A, was first obtained for the relatively simple case of the (zero energy) scattering of a particle by a static central potential which is not sufficiently attractive to bind the particle ${ }^{l}$. Using the Hylleraas-Undheim theorem ${ }^{5}$, it proved to be possible ${ }^{3}$ to extend the method to the scattering of a particle by a static central potential (henceforth to be denoted simply as one body scattering) when bound states do exist. (The interaction will the electromagnetic field is assumed to have been tumed off, so that capture can not take place.) The generalization to the scattering of one compound system by another, for zero initial relative kinetic energy of the two scattering systems, was trivial ${ }^{2}$. The method was then extended to treat positive energy scattering ${ }^{4}$. The presentation was there restricted to the one body problem, taking into account bound states when they exist. The quantity bounded from above in this case is $(-k \cot \eta)^{-1}$, where $\eta$ is the phase shift; this of course reduces to $\mathrm{A}$ as $\mathrm{k}$ goes to zero. Unlike the situation at zero energy, it was unfortunately necessary to restrict the potentials to those which vanish identically beyond some given point, $R$. While the rigorous bounds obtained do not then generally apply directly to the real problem of interest (account must be taken of the truncated portion of the potential) it should be emphasized that the effects of the artificial restrictions on the potentials may be made to be quite small; indeed in 
principle we may come as close as desired to the true problem, by choosing the point $R$ beyond which the potential must varish to be further and further out. In practice the necessary labor increases as the point is moved out, but it increases sufficiently slowly so that it should ordinarily be possible to choose the point $R$ far enough out so as not to have introduced any serious truncation error without having unduly increased the work required.

It is the purpose of the present paper to provide the further extension to the problem of the positive energy scattering of one compound system by another. (For the one channel scattering with which we are presently concerned, this is the final possible extension.) As for positive energy one-body scattering, the various potentials must be truncated.

In the extension of the formalism for positive energy scattering from the one body problem to the case of compound system scattering certain new features arise which were not present in the zero energy case and which require some study. The development of a bound in the method that we have used always effectively involves the expansion of the difference function, the difference between the trial function and the exact function, in terms of some complete set of functions. (Equivalently, the question is always whether or not the difference function satisfies the boundary conditions which are necessary if it is to be possible to use it as one of the trial functions in the application of the Hylleraas-Undheim theorem.) For one body scattering, it was shown ${ }^{4}$ that the complete set coula be taken to be the eigenfunctions either of an associated potential strength eigenvalue problem or of an associated energy eigenvalue problem. The former was found to be preferable, but both gave rigorous bounds. It will be demon- 
strated in Sec. 4 that the potential strength eigenvalue approach admits of a straightforward generalization to include the case of compound system scattering. The energy eigenvalue approach, on the other hand, will not in general be applicable for compound system scattering. It is possible to make the energy eigenvalue approach applicable, but unfortunately only by introducing certain rather restrictive boundary conditions on the trial function. (See Sec. 5). The origin of the necessity of these restrictive conditions, for many body scattering, lies in the fact that for any given $R$, the wave functions of the virtually excited states, while decaying, have not vanished identically.

The technique for getting bounds on phase shifts using the associated potential strength eigenvalue problem was first given by Kato for the one body problem ${ }^{6}$ and later extended to some restricted cases of scattering by compound systems ${ }^{7}$; in this latter work numerical calculations for $\mathrm{e}^{+} \mathrm{H}$ scattering were included. We note that using the method of the present paper it would now be quite feasible to perform the $e^{+H}$ calculation for higher values of the kinetic energy of the incident positron.

It might be noted that the same remarks are applicable for compound system scattering with regard to the error introduced by truncation as were applicable for one body scattering. As examples, consider the scattering of electrons or positrons by Hydrogen. An estimate based on the method of the present paper is that for $R$ of the order of 15 Bohr radii, only about three or four eigenstates need by accounted for ${ }^{8}$, right up to the threshold energies for inelastic scattering, i.e., $7 \mathrm{ev}$ for $\mathrm{e}^{t_{H}}$ and 10 ev for $e^{-} \mathrm{H}$. 
The formal development of a bound is based on a consideration of the associated potential strength eigenvalue problem noted above. One here encounters the necessity of having a definition of the phase shift which is unambiguous. This is in contrast with the nomal requirements where one need only know some trigonometric function of $\eta$, that is, one need merely know $\eta$ modulo $\pi$. Despite the fact that theorems have been surmised which involve the value of $\eta$ (not simply of $\eta$ modulo $\pi$ ), and despite the fact that phase shift values are often discussed for scattering by compound systems, to our knowledge no definition of $\eta$ has been given which is applicable to general compound system scattering. While the above theorems and values undoubtably have some meaning ${ }^{9}$, and certainly so within the context of some approximation, such as the static approximation, it is clear that an unambiguous definition of the phase shift is very much called for. One would of course like to choose a definition which is the most natural possible generalization of the definition for one body scattering, but this general question doesn't arise in our present concern in obtaining a bound on $\cot \eta$.

We now propose a definition of the phase shift for the scattering of one compound systems by another. (As always in the present paper, we are concerned with systems and energies for which the open channels can be decomposed and analyzed in terms of uncoupled channels.) To avoid irrelavant kinematical complications we give the definition for the particular case of the zero orbital angular momentum scattering of a neutron by a nucleus of angular momentum $I$. We further assume, purely for convenience, that the total angular momenturn $J$, and its $z$ projection, $J_{z}$, satisfy 
$J=J_{Z}=I+\frac{1}{2}$. (In sec. 4 the description of the method for obtaining a bound on $k$ cot $\eta$ will be given in terms of this system.) We define the function

$$
g\left(q_{i}\right)=\int F_{i} x_{\frac{11}{22}}(i) \Psi d \tau_{i}
$$

where $q_{i}$ is the distance between neutron $i$ and the center of mass of the nucleus which consists of all the particles except neutron $i . F_{i}$ is the ground state wave function of this nucleus, $\Psi$ is the full scattering wave function, and $x_{\mathrm{SS}_{\mathrm{z}}}(i)$ is the spin function for neutron $i$. The integral is over all coordinates except $q_{i}$, and is understood to represent a summation over all spin indices as well. The phase shift is defined by treating $g\left(q_{i}\right)$ as a one body wave function and applying one of the standard definitions of the phase shift for static potential scattering, namely

$$
\eta=\lim _{m \rightarrow \infty}\left(m \pi-k q_{i}(m)\right)
$$

where $q_{i}(m)$ is the m'th zero in $g\left(q_{i}\right)$.

We note that from the relation

$$
\Psi \rightarrow \operatorname{const} x_{\frac{1}{2} \frac{1}{2}}(i) F_{i} \sin \left(k q_{i}+\eta\right) / q_{i}
$$

it follows that $g\left(q_{i}\right) \rightarrow$ const $\sin \left(k q_{i}+\eta\right)$ as $q_{i} \rightarrow \infty$ which guarantees that $\eta$ modulo $\pi$ as lefined by $\mathrm{Eq} \cdot(2.1)$ is correct.

An Application: Triplet Electron Hydrogen Scattering

It has been stressed that for the purposes of obtaining a bound, the question of the justification of our definition of the phase shift does not 
arise. However, since the question of a useful definition of the phase shift for compound system scattering is a very interesting one in its own right, it may be worth noting that the definition given enables us to see, in a few simple cases at least, how contributions to the zero energy phase shift arise by virtue of the Pauli principle. (Such contributions have been discussed previously ${ }^{10}$ on the basis of an approximate model, the so-called static, or no-polarization approximation.) As an example we consider the zero energy scattering of electrons by atomic hydrogen in the triplet (spatially anti-symmetric) case. We wish to show that the antisymmetry of the wave function implies that the phase shift is at least $\pi$. According to our definition, as applied to this problem, we need only show that the function

$$
g\left(q_{1}\right)=\int_{0}^{\infty} q_{2}{ }^{2} d q_{2} \int_{0}^{\pi} \sin \theta_{12} d \theta_{12} F\left(q_{2}\right) \Psi\left(q_{1}, q_{2}, \theta_{12}\right)
$$

has at least one node. Here $q_{1}$ and $q_{2}$ are the magnitudes of the electron position vectors, $\theta_{12}$ is the angle between those vectors and $F\left(q_{2}\right)$ is the hydrogenic ground state function. (The subscript on $F$ is redundant in this particular discussion and will not be retained here.) The spins have been accounted for. We form the integral

$$
\begin{gathered}
\int_{0}^{\infty} F\left(q_{1}\right) g\left(q_{1}\right) q_{1}{ }^{2} d q_{1}=\int_{0}^{\infty} q_{1}{ }^{2} d q_{1} \int_{0}^{\infty} q_{2}{ }^{2} d q_{2} \int_{0}^{\pi} \sin \theta_{12} d \theta_{12} \\
\quad \times F\left(q_{1}\right) F\left(q_{2}\right) \Psi\left(q_{1}, q_{2}, \theta_{12}\right) .
\end{gathered}
$$


This integral clearly vanishes since $\Psi$ is antisymetric in the electronic coordinates while $F\left(q_{1}\right) F\left(q_{2}\right)$ is symmetric. The fact that the ground state function $F\left(q_{1}\right)$ is nodeless leads to the desired result, namely $\eta \geqq \pi^{11}$.

After the present work was completed, we learned from Dr. A Temkin that he has considered the identical definition of the phase shift for compound system scattering, and further that he has obtained the identical result for the zero energy triplet $\mathrm{e}^{-} \mathrm{H}$ phase shift.

\section{An extension of the triplet $e^{-}$II phase shift result to positive} energy scattering is discussed in sec. $4 . B$.

\section{3 - DEFINITION OF THE PROBLEM FOR WHICH THE BOUND}

IS TO BE OBTAINED

For purposes of clarity we begin with the case in which the mass of one of the scattering systems can be effectively taken to be infinite. A prototype problem would then be the scattering of an electron by a neutral atom of atomic number $Z$. To describe the assumed potentials, we first define three regions in configuration space. The first region contains that portion of configuration space for which all of the $\mathrm{Z}+\mathrm{I}$ electrons are within a sphere of radius $R$ centered about the nucleus of the atom. Region (2) is further subdivided into $\mathrm{Z}+1$ parts; region (2i) contains that portion of space for which all but the $i$ 'th electron are within the sphere of radius $R$, while the $i$ 'th electron is not, where $i$ runs from 1 through $Z+I$ 。 Region (3) consists of the rest of space, that is, the part for which two or more electrons are outside of the sphere.

In region ( 1 ), the potentials are the true (in this case, coulomb) potentials. In region ( $2 i)$, all but the $i$ 'th electron interact as before, 
but the $i$ 'th electron is assumed not to interact with the others. In region (3), the potentials are taken to be infinitely repulsive.

We return now to the particular problem discussed in sec. 2, nanely, the zero orbital angular momentum scattering of a neutron by a nucleus consisting of $Z$ protons and $N$ neutrons. We again divide space into 3 regions, but because the center of mass is not now fixed it is necessary to introduce the auxiliary parameters, $S_{i}$. These are defined, for any distribution of particles, as the radius of the smallest sphere whose center is at the center of mass of the $N+Z$ particles excluding neutron $i$ and which contains all these $N+Z$ particles. ( $i$ of course now runs from $Z+I$ through $\mathrm{Z}+\mathrm{N}+1$.$) The three regions are chosen to be$

(1) $q_{i}<R$

$S_{i}<[(N+Z-I) /(N+Z+I)] R$ for each neutron

(2i) $q_{i}>R$ $S_{i}<[(N+Z-1) /(N+Z+1)] R$ $i=Z+1, Z+2, \ldots$ or $Z+N+1$

(3) the rest of configuration space.

The limit on $S_{i}$ has been chosen such that the $N+1$ regions which make up region (2) are non-overlapping.

The potentials are assumed to satisfy the following requirements. In region (1) the particles interact via two body central potentials which allow for space and spin exchange. As discussed in ref. 4 we exclude tensor forces since we are restricting ourselves to one channel processes, and we are considering non-zero scattering energies. In region ( $2 i$ ) the potentials are of the same form as in (1) except that the interaction between neutron $i$ 
and the rest of the system vanishes. The potentials are taken to be infinitely repulsive in region (3).

It will now be obvious how to define the problem for other systems, such as the scattering of one compound system by another ${ }^{12}$, where each nucleus may carry a net charge ${ }^{4}$.

As shown in ref. 2 the generalization of the Kato identity for the problem under consideration takes the form

$$
\begin{aligned}
\mathrm{k} \cot (\eta-\theta)= & \mathrm{k} \cot \left(\eta_{t}-\theta\right)+\left(2 \mu / \hbar^{2}\right) \int \Psi_{t}^{*}(H-E) \Psi_{t} d \tau \\
& -\left(2 \mu / \hbar^{2}\right) \int \Omega^{*}(H-E) \Omega \alpha \tau,
\end{aligned}
$$

where now the wave function, $\Psi$, which is a solution of $(H-E) \Psi=0$, satisfies the boundary conditions

$$
\begin{aligned}
\Psi=(-I)^{i}(N+I)^{-\frac{1}{2}} x_{\frac{1}{2} \frac{1}{2}}(i) & \left\{F_{i} \sin \left(k_{i}+\eta\right) /\left[q_{i} \sin (\eta-\theta)\right]\right. \\
& \left.+\sum \alpha_{\alpha}^{a} F_{i}(\alpha)_{i}(\alpha)\left(q_{i}\right)\right\}, \\
& \text { in region }(2 i), Z+I \leqq i \leqq Z+N+1, \\
\Psi=0 \quad & \text { in region (3). }
\end{aligned}
$$

Here $\theta$ satisfies $0 \leqq \theta<\pi$ but is otherwise arbitrary. E is the totaI energy, the sum of the relative kinetic energy and of $\mathrm{E}_{\mathrm{g}}$, where $\mathrm{E}_{\mathrm{g}}$ is the ground state energy of the target nucleus. The incident relative wave number, $k$, therefore satisfies 


$$
\hbar^{2} k^{2} / 2 \mu=E-E_{B}
$$

The $\mathrm{F}_{i}(\alpha)$ represent normalized excited state wave functions of the system which does not include the $i$ 'th particle, while the $f_{i}(\alpha)$ are free particle decaying functions. If' for example, $\alpha$ denotes a nuclear state with zero total angular momentum, then

$$
f_{i}(\alpha)=e^{-\kappa_{\alpha} q_{i}} / q_{i}
$$

where

$$
-k_{\alpha}^{2} \hbar^{2} /(2 \mu)+E_{\alpha}=E
$$

with $\mathrm{E}_{\alpha}$ representing the energy of the $\alpha^{\prime}$ th nuclear excited state. If $\alpha$ represents a nuclear state with angular momentum $L$, then $F_{i}(\alpha)_{i}(\alpha)$ will involve a sum of products, each containing a pair of angular momentum eigenfunctions. $\Psi_{t}$ satisfies similar boundary conditions with $\eta$ and $a_{\alpha}$ replaced by $\eta_{t}$ and $a_{\alpha t}$, respectively. It is our purpose to obtain a lower bound on the error integral, $\int \Omega^{*}(H-E) \Omega d \tau$, where $\Omega \equiv \Psi_{t}-\Psi$, thereby providing an upper bound on $k \cot (\eta-\theta)$.

$$
4-\text { BOUND ON } \mathrm{k} \cot (\eta-\theta) \text { and on } \eta
$$

\section{A - The Associated Potential Strength Eigenvalue Problem} We consider the equation

$$
(H-E) \Phi=\mu \rho \Phi
$$

where $\rho$ is positive, but otherwise arbitrary, in region (1) and vanishes 
elsewhere. $\Phi$ satisfies the boundary conditions

$$
\begin{aligned}
& \Phi=(-I)^{i}(N+1)^{-\frac{1}{2}} x_{\frac{11}{2}}(i)\left\{\text { const } F_{i} \sin \left[\mathrm{kq}_{i}+\delta(\mu)\right] / \mathrm{q}_{i}\right. \\
& \left.+\sum_{\alpha} b_{\alpha} F_{i}^{(\alpha)} f_{f_{1}}(\alpha)\left(q_{1}\right)\right\} \\
& \text { in region (2i), } Z+I \leqq i \leqq Z+N+I \text {, } \\
& \Phi=0 \quad \text { in region (3). }
\end{aligned}
$$

The eigenfunctions $\Phi_{n}$ and their corresponding eigenvalues $\mu_{n}$ are defined by the condition ${ }^{13}$

$$
\delta\left(\mu_{n}\right)=\theta+n \pi
$$

In the following, we make the reasonable assumption that for finite $\mu$ and for positive scattering energies $\delta(\mu)$ is a continuous function of $\mu$; this is of course known to be true in the one body problem.

In order to apply the Hylleraas-Undheim theorem, which leads to the desired bound, it is necessary to establish the existence of a lowest eigenvalue. By virtue of the monotonicity of $\cot \delta(\mu)$ the continuity of $\delta(\mu)$ leads to the monotonicity of $\delta(\mu)$ itself with respect to $\mu$. We therefore need only show that $\delta(-\infty)$ is finite. In fact, as in the one body case, we have $\delta(-\infty)=-k R$. Thus, for $\mu=-\infty$ the corresponding wave function, $\Phi(-\infty)$, vanishes in region (1) and has the form given in Eqs. (4.1) in regions (2) and (3). According to our definition of the phase shift (see Sec, 2), $\delta(-\infty)$ is determined by examining the function 


$$
g\left(q_{i},-\infty\right)=\int F_{i} x_{\frac{11}{22}}(i) \Phi(-\infty) d \tau_{i} .
$$

We clearly have

$$
\begin{array}{rlrl}
g\left(q_{i},-\infty\right) & =0 & , & q_{i}<R \\
& =\text { const } \sin \left[k q_{i}+\delta(-\infty)\right] / q_{i}, & q_{i} \geqq R
\end{array}
$$

from which the stated value of $\delta(-\infty)$ is immediately deduced.

The boundary conditions satisfied by the difference function, $\Omega$, are given by

$$
\begin{array}{ll}
\Omega=(-1)^{i}(N+1)^{-\frac{1}{2}} x_{\frac{11}{22}}\left\{\operatorname{const} F_{i} \sin \left(k q_{i}+\theta\right) / q_{i}\right. \\
& \left.+\sum_{\alpha} c_{\alpha} F_{i}(\alpha)_{f_{i}}(\alpha)\left(q_{i}\right)\right\}, \\
& \text { in region }(2 i), Z+I \leqq i \leqq z+\mathbb{N}+1 \\
\Omega=0 & \text { in region (3), }
\end{array}
$$

which is of the form satisfied by the eigenfunctions $\Phi_{n}$. Therefore, $\Omega$ is an allowable trial function in an application of the Hylleraas-Undheim theorem to the associated potential strength eigenvalue problem. The formal steps required to derive an upper bound on $k \cot (\eta-\theta)$ are then identical to those described in ref. 4. The upper bound obtained, the basic result of the present paper, is

$$
\begin{aligned}
k \cot (\eta-\theta) \leqq k & \cot \left(\eta_{t}-\theta\right)+\left(2 \mu / \hbar^{2}\right) \int \Psi_{t}{ }^{*}(H-E) \Psi_{t} \mathrm{~d} \tau \\
-\left(2 \mu / \hbar^{2}\right) & \sum_{n=-P}^{-P+T^{\prime}-1} \frac{1}{\mu_{n t}}\left|\int \Phi_{n t}{ }^{*}(H-E) \Psi_{t} d \tau\right|^{2},
\end{aligned}
$$


where the $\Phi_{n t}$ are trial functions which satisfy the boundary conditions

$$
\begin{aligned}
& \Phi_{n t}=(-1)^{1}(N+1)^{-\frac{1}{2}} x_{\frac{11}{2} \frac{1}{2}}(1)\left\{\text { const } F_{1} \sin \left[\mathrm{kq}_{1}+\theta\right] / q_{1}\right. \\
& \left.+\sum_{\alpha} b_{\alpha t_{1}}{ }^{(\alpha)} f_{1}(\alpha) \underset{m}{\left(q_{1}\right)}\right\} \\
& \text { In region ( } 21), \mathrm{Z}+\mathrm{I} \leqq 1 \leqq \mathrm{Z}+\mathrm{N}+\mathrm{I} \\
& \Phi_{n t}=0 \quad \text { in region (3) }
\end{aligned}
$$

as well as the conditions

$$
\begin{aligned}
& \int \Phi_{n t}^{*} \Phi_{m t} \rho d \tau=\delta_{n m} \\
& \int \Phi_{n t}^{*}(H-E) \Phi_{m t} d \tau=\mu_{n t} \delta n, \quad \mu_{n t}<0 .
\end{aligned}
$$

We here have the condition that $\theta-(P+1) \pi \leqq-k R<\theta-P \pi$. Note that the conditions $\mu_{n t}<0$ impose some minimum requirements of accuracy on the trial functions $\Phi_{n t}$. The inequality of $\mathrm{Eq} \cdot(4.2)$ is valid provided that the number of trial functions, T', which have been found with the required properties is equal to the exact (unknown) number, $T$, of elgenfunctions with negat1ve elgenvalues. In almost every case, however, it should be possible on the basis of the numerical calculations involved in the determination of the negative trial elgenvalues $\mu_{n t}$ to be falrly certain that $T^{\prime}$ does in fact equal $\mathrm{T}$.

\section{$B$ - The Conditional Inequality}

In the one body case, it was shown ${ }^{4}$ that by the use of the conditional Inequal1ty ${ }^{14}$ one could obtain a rigorous bound on $\eta$ even when one could not be sure that $T^{\prime}=T$, that 1 , even when one did not have a rigorous bound on $\mathrm{k} \cot (\eta-\theta)$. It was found that

$$
\eta>\eta_{L}
$$


where $\eta_{\mathrm{L}}$ ' is defined by the equations

$$
\begin{aligned}
& \mathrm{k} \cot \left(\eta_{L}{ }^{\prime}-\theta\right)=\text { right hand side of the one body equivalent of } \mathrm{Eq} \cdot(4 \cdot 2) \\
& \mathrm{T}^{\prime} \pi-\mathrm{kR}<\eta_{\mathrm{L}}{ }^{\prime}<\left(\mathrm{T}^{\prime}+1\right) \pi-\mathrm{kR} \text {. }
\end{aligned}
$$

This lower bound on $\eta$, while rigorous, will be too low by roughly $\left(T^{-} T^{t}\right) \pi$ if $T^{\prime}$ is less than T. ( $T^{\prime}$ can never be greater than $T$, for we can never find more negative potential strength eigenvalues than actually exist.) The lower bound then obtained will then be useless with regard to a comparison with the experimental data, though it may still be useful as the starting point for further theoretical calculation ${ }^{14}$.

The above developments can be taken over directly, with complete rigor, for the many body case. There is nevertheless one respect in which the use of the conditional inequality differs for the many body problem when identical particles are present from its use for the one body scattering problem. Thus, account must be taken of the additional nodes which arise from the symmetry requirements on the wave function, or the lower bound, 'IL', while rigorous, may be too low by a multiple of $\pi$ even when the number of eigenstates with negative eigenvalues $\mu_{n}$ has been correctly accounted for. The effect of the Pauli principle can perhaps be better understood through a comparison of one body scattering and the triplet (spatially antisymetric) scattering of electrons by hydrogen atoms. For both systems, we have that $\delta(-\infty)=-k R$. [For $\mu=-\infty$, the one body scattering function vanishes for $0 \leqq q \leqq R$, as does the equivalent one body scattering function, $\left.g\left(q_{i}\right) \cdot\right]$ However, while $\delta(\mu)$ is a continuous function of $\mu$ in the neighborhood of $\mu=-\infty$ for one body scattering, it is not continuous for $\mathrm{e}^{-} \mathrm{H}$ triplet scattering. It is to be recalled that the $e^{-} \mathrm{H}$ problem under consideration 
is the true problem modified by the introduction of a cutoff and a repulsive barrier. The proof given in sec. 2 that $\eta \geqq \pi$ at zero energy can then be immediately extended for $k \neq 0$ to show that $k R+\eta>\pi$. To see this, we note that in the definition of $g\left(q_{1}\right), E q .(2.2)$, the range of integration, 0 to $\infty$, can be replaced by $O$ to $R$ since $F\left(q_{2}\right)$ exists only in that range. It then follows, using the same symmetry argument as was used at zero energy, that the integral

$$
\begin{aligned}
& \int_{0}^{R} F\left(q_{1}\right) g\left(q_{1}\right) q_{1}{ }^{2} d q_{1}= \\
& \quad \int_{0}^{R} q_{1}{ }^{2} d q_{1} \int_{0}^{R} q_{2}^{2} d q_{2} \int_{0}^{\pi} \sin \theta_{12} d \theta_{12} F\left(q_{1}\right) F\left(q_{2}\right) \Psi\left(q_{1}, q_{2}, \theta_{12}\right)
\end{aligned}
$$

vanishes. It follows that $g\left(q_{1}\right)$ has a node for some $q_{I}<R$, which in turn, as has been previously shown ${ }^{7}$, implies the inequality $k R+\eta>\pi$. The argument is independent of the form of the potential so that we have, more generally, $\mathrm{kR}+\delta(\mu)>\pi$ for all finite $\mu$, and in particular, for $-\infty<\mu<\mu_{-\mathrm{P}}$, where $\mu_{-\mathrm{P}}$ is the lowest eigenvalue. Accordingly, in the application of the conditional inequality technique to this problem, we would replace

$$
T^{\prime} \pi-k R \leqq \eta_{L}^{\prime} \leqq\left(T^{\prime}+I\right) \pi-k R,
$$

by the form

$$
\left(T^{\prime}+I\right) \pi-k R \leqq \eta_{L}^{\prime} \leqq\left(T^{\prime}+2\right) \pi-k R,
$$

which gives rise to a more accurate lower bound on $\eta$. 
5 - THE USE OF ENERGY EIGENFUNCTIONS

It was shown in ref. 4 that in the one body case the introduction of an associated energy eigenvalue problem will lead to similar though less general results than obtained using the associated potential strength eigenvalue problem. When the scatterer is a compound system, however, the energy eigenvalue approach is not valid, since by definition the energy eigenfunctions vanish at the box [i.e., at the boundary of region (I)], while the difference function, $\Omega$, does not; $\Omega$ is therefore not an allowable trial function in the application of the Hylleraas-Undheim theorem. In the one body problem, the difference function, there denoted by w, does vanish at the box. There, the form of $w$ is known to be

$$
w(r)=\text { const } \sin (k r+\theta), \quad r \geqq R,
$$

so that by the appropriate choice of $R$, it is a trivial matter to arrange to have $\mathrm{w}(R)=0$. In compound system scattering, however, $\Omega$ contains, in addition to a term of the above form, contributions from the virtually excited states, with unknown amplitudes. There is then no choice of $R$ which will cause $\Omega$ to vanish, though clearly one can still arrange to have the non-decaying component of $\Omega$ vanish at the edge of region (1).

It is of course true that by choosing $R$ large enough, it is possible to make the effect of the decaying states quite small. However, there 1s a reason for not making $\mathrm{R}$ too large; there are then too many energy eigenstates which must be accounted for in the determination of a bound. Furthermore, one does not really know tow large $R$ must be made before the effect of the decaying states is truly small. Not only does one then have an approximate if well defined problem (the potentials having been truncated), 
but one has an 'approximate bound' on that approximate problem.

Recently, Percival 15 has attempted to extend a technique ${ }^{16,17}$

introduced for obtaining a bound on the phase shift for one body scattering to the compound system scattering case. The approach was restricted to the very special case where no Pauli exchange can take place between particles in the scattered and scattering systems, but even for this extremely restrictive case, we can find no rigorous justification for this approach. This is not very surprising, since we have just seen that the straightforward energy eigenvalue approach does not lead to a rigorous bound, and, as indicated in ref. 4 , there is an intimate connection for the one body case between the energy eigenvalue approach and that of Percival and of Risberg. It seems worthwhile nevertheless to give some details as to why the Percival approach is not rigorous for many body scattering.

It will be recalled that in one body scattering, there is a simple connection between the wave function for the eigenstate with energy $E_{j}$ of a particle confined to the region $R$ within which $V(r)$ exists and the scattering wave function at the energy $\mathrm{E}_{j}$ for the potential $\mathrm{V}(r)$ truncated at $R$; the scattering wave function is simply the extension of the eigenstate wave function, continuous in value and slope at $R$. There is no such simple connection in the case of compound system scattering. Once again, the origin of the difference is the existence of virtually excited states, for a wave function which vanishes at the edge of region (1) must contain terms of the form

$$
F_{i}(\alpha)\left(e^{-k_{\alpha}\left(q_{i}-R\right)}-e^{+\kappa_{\alpha}\left(q_{i}-R\right)}\right) / q_{1},
$$


where for simplicity we consider the case for which $\mathrm{F}_{i}(\alpha)$ represents a nuclear state of zero total angular momentum. Due to the presence of the exponentially increasing term, the smooth continuation of the eigenstate wave function into the rest of configuration space cannot represent a scattering wave function.

It is true that for R "Iarge enough", the approach is not unreasonable, and that is really all that Percival claims, but one does not obtain a rigorous bound on any well defined problem.

It may be of some interest that the results of the present paper can be used to show that there exists a modification of the Percival approach which can be justified. The modification is not a trivial one since it involves placing an additional restriction on the trial functions. This modified form, however, being a special case of our general development, has the interesting property that it does include the effects of the Pauli principle.

Thus, suppose we choose for $\rho$ some constant value in region (1). (It must of course vanish elsewhere.) Furthermore, we choose the boundary conditions satisfied by the trial function, $\Psi_{t}$, to be of the form

$$
\begin{aligned}
& \Psi_{t}=(-I)^{i}(N+I)^{-\frac{1}{2}} x_{\frac{1}{2} \frac{1}{2}}(i)\left\{F_{i} \sin \left(k q_{i}+\eta_{t}\right) /\left[q_{i} \sin \left(\eta_{t}-\theta\right)\right]\right\} \\
& \text { in region ( } 2 i) \quad Z+I \leqq i \leqq Z+N+I \\
& \Psi_{t}=0 \quad \text { in region (3); }
\end{aligned}
$$

that is, no sum over excited states appears. Similarly, we choose the trial 
eigenfunctions, $\Phi_{n t}$, such that the coefficients of the terms in the sum over excited states vanish. With the choice $k R+\theta=m \pi, m$ an integer, the functions $\Phi_{n t}$ vanish at the boundary of region (1), i.e., they are allowable trial energy eigenfunctions in region (1). Indeed, with our choice of $\rho$ the boundary conditions along with the conditions given by Eqs. (4.4) are just those placed on trial energy eigenfunctions in the application of the Hylleraas-Undheim theorem. The resultant inequality for $\mathrm{k} \cot (\eta-\theta)$, along with ar application of the conditional inequality, then leads to a modification of the Percival result which is rigorous and which does allow for identical particles. However, since one here requires the use of trial functions whose flexibility is restricted by the physically unnatural conditions mentioned above (the exact function does not satisfy those conditions) there is a limitation on the accuracy of the bound that can be obtained. In fact, it may well not be possible to find the appropriate number of trial functions $\Phi_{n t}$ of the prescribed restricted form (i.e., we might necessarily have $\mathrm{T}^{\prime}<\mathrm{T}$ ) in which case the bound obtained on $\eta$ will be too low by approximately $\left(T-T^{\prime}\right) \pi$.

It should be emphasized that while we obtain valid results using (restricted) trial energy eigenfunctions the difference function, $\Omega$, cannot be expanded in terms of energy eigenfunctions. The difficulty has been bypassed by the use of the associated potential strength eigenvalue problem as the starting point. The point is that in this latter approach one places conditions only upon the oscillatory term, through having specified the phase shift $\delta\left(\mu_{n}\right)$; no restrictions are placed on the amplitudes of the virtually excited decaying states. 
The primary purpose of the present paper is the derivation of Eqs. (4.2), (4.3), and (4.4). The analysis of previous work presented in this section is simply a byproduct. We have presented the analysis for two reasons. Firstly, it sheds some light on the methods of the present paper as well as on previous work. Secondly, there will be occasions when for practical reasons one would use trial functions of the restricted form; that is what was done, for example, in the analysis of the scattering of positrons by Hydrogen atoms ${ }^{7}$. 
References

1. I. Spruch and L. Rosenberg, Phys. Rev. 116, 1034 (1959).

2. L. Spruch and I. Rosenberg, Phys. Rev. 117, 1095 (1960).

3. L. Rosenberg, L. Spruch, and T.F. O'Malley, Phys. Rev. 118, I84 (1960).

4. L. Rosenberg and L. Spruch, Phys. Rev. 120, 000 (1960). There is a typographical error in the second equation before Eq. (5.4a). $\delta(\infty)$ shoula be $\delta(-\infty)$.

5. E.A. Hylleraas and B. Undheim, Z. Physik 65, 759 (1930).

ó. T. Kato, Progr. Theoret. Phys. (Kyoto) 6, 395 (1951).

7. L. Spruch and I. Roseriberg. Phys. Rev. 117, .143 (1960).

8. This estimate was arrived at with the aid of a rather crude approximation, namely, by assuming that the incoming particle is unaffected by the Hydrogen atom. It is nevertheless adequate for the purpose intended, i.e., to indicate that there exists a significant range of energies in which the calculation can be performed without an undue amount of labor. Of course the actual bound obtained will not depend on any such approximation.

9. It seems likely, for example, that when finally some definition will have been introduced of sufficient utility to be generally acceptable, the phase shifts that follow from this definition will generally be the same as those that have been quoted.

10. P Swan, Proc. Roy. Soc. A. 228, 10 (1955).

11. The zero energy triplet phase shift is generally taken to be $\pi$. We note that this has only been established within the context of the static approximation. Indeed, as observed above, no definition of the phase shift for the true problem has previously been advanced. 
12. In this case, incidentally, the definition of a one body scattering function, in terms of which the phase shift is defined, will require that we multiply the true scattering function by the product of the ground state wave functions of the isolated systems before performing an integration.

13. The fact that the Hamiltonian, H, is Hermitian under the assumed boundary conditions for the $\Phi_{n}$ is easily verified; the required calculation is quite similar to the one by which the Kato identity was established (see ref. 2) and we omit the detials.

14. I. Spruch, Phys. Rev. 109, 2149 (1958).

15. I.C. Percival, Phys. Rev. 119, 159 (1960).

16. I.C. Percival, Proc. Phys. Soc. (London) 70,494 (1957).

17. V Risberg, Arch. Math. Naturvid. 53, I (1956). 
NEW YORK UNIVERSITY

INSTTTUTE OF MATHEMATICAL SCIENCES LIBRARY

R5 Waverly Place, New York 3, N. Y.

\section{APR 251961}

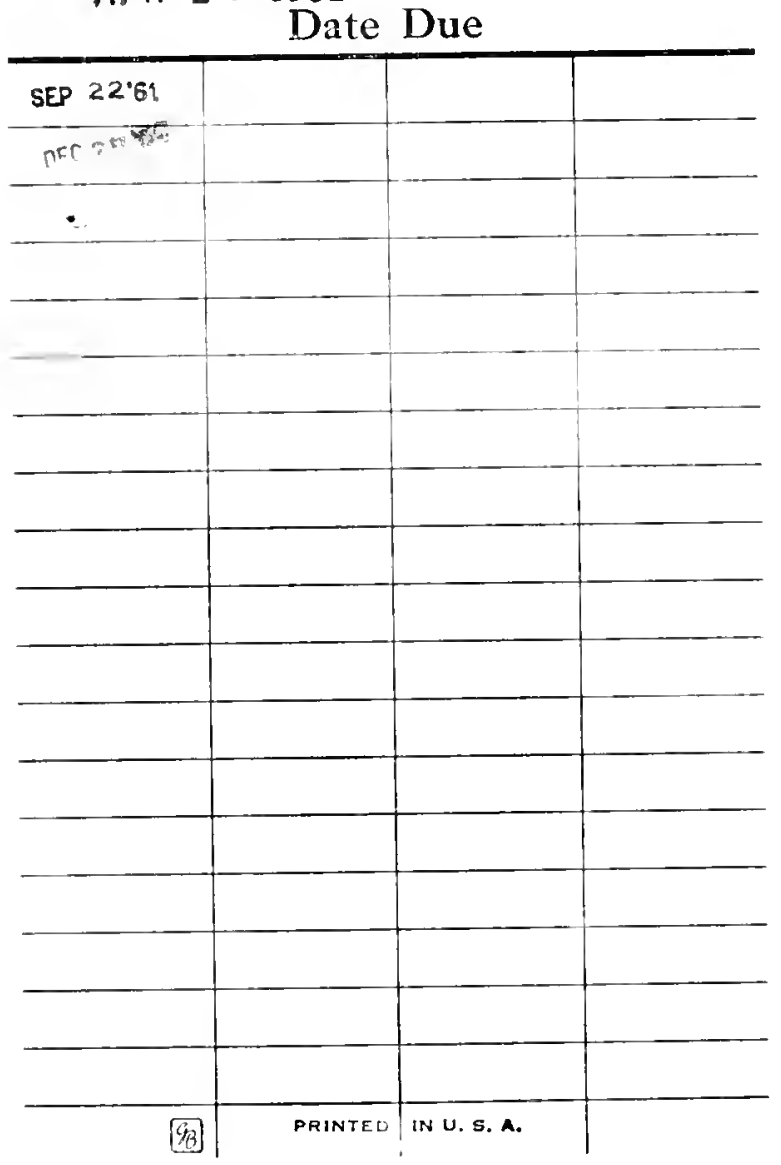


INSTITUTE OF MATHEMATICAL SCIENCES

LIBR,ARY

25 Waverly Place, New York 3, N. Y.

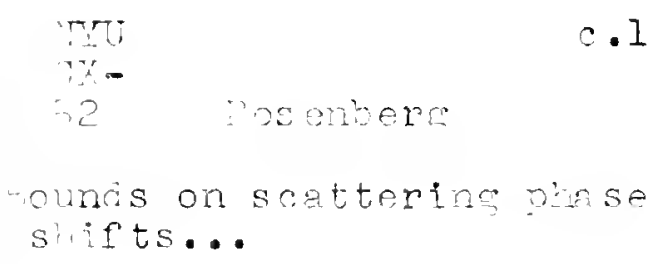

N. Y. U. Institute of Mathematical Sciences

25 Waverly Place

New York 3, N. Y. 
